\title{
Labor Productivity and Obsolete Skills in a Growth Model with Production by Layers
}

\author{
Orlando Gomes *, \\ Instituto Superior de Contabilidade e Administração de Lisboa (ISCAL/IPL) \\ and Instituto Universitário de Lisboa (ISCTE-IUL), Business Research Unit (UNIDE)
}

- Version 3, June 2013

\section{Structured abstract}

Purpose: This paper studies growth dynamics in a model where labor productivity is shaped by two forces. On one hand, it is determined by the extent in which available technology has been already explored. On the other hand, some labor skills may become obsolete, jeopardizing the ability of the labor input in creating value, namely when a transition between technological states takes place.

Design/methodology/approach: A theoretical model is developed, based on previous work about hierarchical organizations of production, in order to build an integrated structure of analysis for growth, productivity, innovation and obsolescence of skills.

Findings: In a setting in which output grows through the accumulation of layers of activity, the generation of income and the evolution of techniques will be determined by the choice of a representative agent, who faces a trade-off between consumption utility and the desire to maintain intact the skills of the labor force.

Research limitations/implications: The theory provides an analytical structure to think about skill acquisition and skill obsolescence in the context of economic growth. Further work is necessary, namely at an empirical level, to test the validity and the reasonability of the model's implications.

Originality/value: The paper sheds light on the role of labor productivity as a growth determinant. It seeks a deeper understanding on the relationship between human capabilities and the efficient use of technology.

Keywords: Labor productivity, Obsolete skills, Hierarchical organization, Economic growth, Technological change, Utility maximization.

JEL classification: O33, O41, J64. Paper type: Research paper.

* Address: Instituto Superior de Contabilidade e Administração de Lisboa (ISCAL/IPL), Av. Miguel Bombarda 20, 1069-035 Lisbon, Portugal. E-mail: omgomes@iscal.ipl.pt

^Acknowledgments: I thank the insightful comments of two anonymous referees, which helped in improving the contents of the paper. The usual disclaimer applies. Financial support from the Business Research Unit of the Lisbon University Institute under project PEst-OE/EGE/UI0315/2011, is also gratefully acknowledged. 


\section{Introduction}

The paper develops a growth model that integrates technological exploitation and innovation, productivity dynamics and the evaluation of preferences relating the preservation of working skills. The basic structure of the model is inspired on the growth through organizations theory of Garicano and Rossi-Hansberg (2010), although a series of adaptations are introduced over that prototype model in order to highlight how the process of economic growth and technological development might shape (and be shaped by) the evolution of aggregate labor productivity in time. $\underline{1}$

Fundamentally, we present a theory that explores the role of labor productivity in the process of wealth accumulation. Capital accumulation (and the accumulation of other physical inputs) is ignored in this particular setting, in order to better highlight how human capabilities respond when faced with changing technological conditions. This approach gains a particular relevance when thinking about the drivers of growth in today's developed world. Most of the growth in economies with already high living standards is based on the efficient use of new techniques and on how the economy can create the environment that serves the dynamic process through which innovations are given their best possible use. Therefore, by adapting a setup of production by layers in order to include skills' creation, use and obsolescence, this paper intends to offer a contribution to understand how developed economies eventually feed a process of continuous and sustained growth.

We approach labor productivity as the result of a tension between two forces: on one hand, as a given technological state is explored, workers gain the incentive

1 Garicano and Rossi-Hansberg (2010) claim that their contribution is twofold: first, it explicitly models learning in organizations by specifying a hierarchy of production by layers that allows to understand how the economy is structured to explore the available technology; second, it focuses on a specific technology, namely information and communication technology (ICT), in order to explain how communication impacts on growth through organizational learning. In the current paper, the discussion on ICT is discarded, and the production by layers setup is, alternatively, used to address the impact of labor productivity on growth. This naturally implies introducing specific changes into the framework, which now considers elements that are absent in the original framework, as it is the case of the possibility of skills preservation in a context where such skills are subject to obsolescence. In the end, new results will emerge, and these go precisely in the direction suggested by Garicano and Rossi-Hansberg (2010, page 651), who state that their contribution should be viewed 'as the start of an effort to understand, at a deeper microeconomic level, the use of the labor input usually introduced in aggregate production functions. What matters for development is not how many units of labor are used, but how these units are organized, and how this changes over time.' 
to acquire additional skills in order to handle such technology; this raises labor productivity, and the initial inefficiency arising from the use of a novel, unknown and not yet explored technology tends to disappear as soon as the available technology becomes of common use.

On the other hand, we will have a share of the labor force that loses its usefulness because the corresponding skills become obsolete; developed tasks become trivial, and they will no longer need human intervention to be successfully undertaken. The argument is that when some of the tasks associated to a technology become common knowledge they can be automated and people are no longer necessary to develop them. This argument leads to the emergence of unproductive labor of a second type: under the current technology, workers are set aside because their skills are no longer required. Workers falling in the skill obsolescence trap can engage in skill acquisition programs that will allow them to be prepared to integrate production and to be potentially productive as soon as a new technological state emerges. This will imply their maintenance in the current production process, although the same activities could be developed without human intervention.

In synthesis, departures from full labor productivity can be split into two realities: one concerning unexplored technology and the other relating skills obsolescence. We will designate these by short-run productivity loss (the one related to yet unexplored technology) and long-run productivity loss (the one associated to skill obsolescence).

The model will involve a process of creative destruction. When a new technology is adopted, the existing organization of production is replaced by another one and the process of solving problems in order to exploit technological resources starts again. The periods that immediately follow a technological breakthrough are necessarily periods of falling productivity, because skills of the old economy / technology are not kept and the new technology has not yet been thoroughly explored. The endogenous nature of the assumed process of technology adoption, that involves an optimal choice by a representative agent, implies that innovation occurs only when the current technological state has been explored in some extent.

Processes of creative destruction have been analyzed in the growth literature since the seminal work of Grossman and Helpman (1991) and Aghion and Howitt (1992). Creative destruction is a useful concept in order to deal with productivity and efficiency issues. Recent literature points to important relations that can be established between entrepreneurship, organizational structure of firms and adaptation to new knowledge, on one hand, and productivity changes, growth and business cycles, on the other; see, for example, Hodgson (1998), Thesmar and Thoenig (2000), Francois and Lloyd-Ellis (2003) and Phillips (2012).

Although founded on technology evolution, our analysis pursues fundamentally the understanding of the link between labor productivity and 
growth. What is the impact of technology evolution on labor productivity? Should one be concerned with a productivity slowdown caused by creative destruction? How much is the society prepared to sacrifice in order to maintain workers holding obsolete skills as a part of the labor force? These are the fundamental concerns in this paper, which are also present in the literature that recently has approached the relation between growth and productivity. For instance, Apergis, Economidou and Filippidis (2008) explore the connection between labor productivity, innovation and technological spillovers in a group of manufacturing industries; these authors conclude that $R \mathcal{E} D$ and human capital accumulation have a direct impact on labor productivity, both via innovation and diffusion of knowledge. This link is also investigated by Cosar (2011), who develops a model where productivity is enhanced when producers hire skilled labor in order to use more efficiently an available exogenously evolving stock of world knowledge. Labor productivity differs across countries; in Razzak (2007) these differences are associated essentially to endogenous technology shocks, more than to other factors, like culture, laws or institutions.

Another recent work that deals directly with the relation between innovation and labor skills is Acemoglu, Gancia and Zilibotti (2012). These authors study how an innovation diffuses given the profile of the available labor input. When introduced, the handling of the new technology requires the use of skilled labor. In order for this technology to become of common use, a costly standardization process must be followed; this process will allow unskilled labor to be able to successfully manage the new techniques. In such setting, standardization can be an engine of growth, in the sense it allows innovation to spread, but it also constitutes a potential barrier to the creation of wealth, since it diverts resources from the production process itself; as a result, it will be possible to determine an optimal speed of standardization. Our approach, although dealing with the same issue of technological exploitation by more or less skilled workers, diverges from the mentioned study on a crucial point: skill acquisition is endogenous; in our setting there is not an a priori separation between skilled and unskilled. Skills acquisition is endogenous in the sense it emerges directly from how the model is designed in order to incorporate the incentives faced by workers.

It is essentially in the context of overlapping generations (OLG) models that the relation between productivity and growth has been theoretically approached. In Nissim (2007), this type of model is used to demonstrate that, as the economy progresses, workers are mobilized up to better jobs, thus increasing overall productivity and making income distribution less uneven. In Teles and Andrade (2008), the OLG setup is used to approach the quality of public education and how this impacts on productivity and growth. From an empirical point of view, a relevant study on the connection between productivity growth and the evolution of aggregate income is offered by Serletis and Feng (2006).

The remainder of the paper is organized as follows. Section 2 characterizes 
how a specific technology is exploited in an economy organized by layers of production. In section 3, the model is adapted in order to account for skills obsolescence and, in section 4 , one addresses how a representative agent choice between consumption and skills preservation is evaluated and how this impacts on production, use of technology and productivity. In section 5, one takes an endogenous process of innovation; a new technology temporarily lowers skills obsolescence but makes short-run productivity losses to re-emerge. Finally, section 6 concludes.

\section{The Exploitation of a Technology}

Take a discrete sequence of time periods, $t=0,1, \ldots$ At the initial date, $t=0$, a new technology, represented by the index $A>0$, is adopted. This section characterizes how technology $A$ is explored from the moment it becomes available to the period when it is replaced by a more advanced technological level. If no other technological advancement takes place in the future, the economy converges to a long-term steady-state in which the available state of knowledge is explored in its full extent.

Basically, this is a framework where a learning process takes place over time: the more consecutive periods are considered, the larger is the percentage of the available techniques that are put to a productive use. In this setup, capital accumulation is ignored, and labor serves one single purpose: to solve the problems associated with the utilization of technical resources. Aggregate output is defined by $Y$ and the potential output will just correspond to the available technology, i.e., $Y_{P}=A$. Effective output, however, will tend to differ from $Y_{P}$ because it takes time to explore the potentialities of technology; this is done through layers of activity and, at each period, a single new layer arises.

Consider that technology $A$ has associated an unspecified number of problems in the interval $[0, z]$. Agents act with the purpose of solving these problems in a sequential order (starting from the most basic and common ones), given a probability distribution function $f(z)$ that is continuous and decreasing. The share of problems the worker will be able to solve is, then, translated on the value of the cumulative distribution function $F(z)$. In other words, agents will successfully address the problems that lie within their knowledge set, which are necessarily a share of the whole of the problems associated with the prevailing technological state.

At the initial date, $t=0$, workers will generate $A F\left(z_{0}\right)$ units of output, in the case in which they solve $z_{0}$ problems associated with $A$. If one conceives that agents are endowed with a unit of effort, one realizes that only a share $F\left(z_{0}\right)$ of this effort gives place to the creation of value; hence, $F\left(z_{0}\right)$ can be interpreted as the productivity level in this first period, for the unique layer of production that is 
initially considered. Productivity in the first layer will be denoted by $e_{t}^{L 1}$, given time period $t$. Productivity is measured in the scale from 0 to 1 , where 1 represents a scenario where the labor force is used with full efficiency. On the other extreme, productivity is zero, i.e., human capabilities are completely wasted.

For $t=0, e_{0}^{L 1}=A F\left(z_{0}^{L 1}\right)$, where $z_{0}^{L 1}$ is the specific amount of problems associated with $A$ that are successfully solved at the initial period by workers in the, for now, only existing layer L1. To compute the optimal value of $z$ at this first stage, one needs to know the cost involved in the acquisition of knowledge; this cost will be represented by $\zeta \geq 0$, so that the total learning costs concerning the acquisition of knowledge are $\zeta z$ at each period.

Workers will solve a static optimization problem; they will maximize their wage by finding an optimal value of $z$. The wage is defined as the difference between the income generated from the available knowledge and the learning costs. The problem is

$$
w_{0}^{L 1}=\max _{z_{0}}\left[A F\left(z_{0}\right)-\zeta z_{0}\right]
$$

In expression (1), $w_{0}^{L 1}$ represents the wage rate workers at layer $L 1$ receive at $t=0$, as the result of solving the maximization problem. The control variable in (1) is $z$; hence, workers weight costs and benefits of acquiring skills in order to manage an optimal quantity of problems $z_{0}^{L 1}$, which is the solution of (1). Once found the value of $z_{0}^{L 1}$ one will be able to explicitly present not only the productivity level, but also the wage rate, $w_{0}^{L 1}=A F\left(z_{0}^{L 1}\right)-\zeta z_{0}^{L 1}$ and the level of effective output, $Y_{0}=A F\left(z_{0}^{L 1}\right)$.

Define a measure of productivity loss as the complement of variable $e_{t}$, which, at this first stage, takes the expression $u_{0}=1-F\left(z_{0}^{L 1}\right)$. Productivity waste exists because, when evaluating benefits and costs of exploring problems associated with a given technical state, workers realize that it is not financially advantageous to acquire the knowledge required to engage in the whole of the productive activities associated with the exploitation of the available technological state.

To further elucidate about the mechanics of the proposed framework, we consider an exponential distribution of problems, such that $F(z)=1-\exp (-\lambda z)$, $\lambda>0$. With this distribution function, it is straightforward to obtain the value of $z$ that solves (1),

$$
z_{0}^{L 1}=-\frac{1}{\lambda} \ln \left(\frac{\zeta}{\lambda A}\right)
$$

The constraint $\quad \zeta<\lambda A$ must apply in order to guarantee that a positive 
number of problems is successfully solved. The corresponding values of productivity, wage and output are

$$
\begin{gathered}
e_{0}^{L 1}=1-\frac{\zeta}{\lambda A} \\
w_{0}^{L 1}=A-\frac{\zeta}{\lambda}\left[1-\ln \left(\frac{\zeta}{\lambda A}\right)\right] \\
Y_{0}=A-\frac{\zeta}{\lambda}
\end{gathered}
$$

The better the technology level and the lower the costs of knowledge acquisition, the higher will be the values of the productivity index, of the wage and of the output level. Observe that the level of output corresponds to potential output minus a constant $\zeta / \lambda$. Productivity loss in this first period, given by $u_{0}=\frac{\zeta}{\lambda A}$, is a short-run and temporary waste of productivity; as new layers of production are added in subsequent periods, this rate will become progressively smaller and will, eventually, converge to zero as the number of layers tends to infinity.

Problems that are not solved at $t=0$ will be freely available to be solved in a second layer of production at $t=1$, by the workers who are not associated with the activity in the first layer. A decisive distinction exists between the problems faced in each of the layers. Because problems at layer L2 are those that agents at layer L1 were unable to solve, the organizational structures of society (i.e., production organizations) realize that a larger incentive has to be attributed to workers in order for these to be willing to engage in the exploitation of more sophisticated problems associated with technology $A$. This idea is translated on a new parameter, $h_{L 2} \in(0,1)$, which will be introduced in the wage maximization problem of workers at $L 2$, and that leads to a larger wage compensation attributed in this second layer, of harder to solve technological challenges. Thus, the above parameter may be interpreted as a prize or a bonus that is offered as a stimulus to workers to incur in higher knowledge acquisition costs, making them better prepared to address more challenging problems on the available technology frontier.

At time $t=1$, agents at layer $L 1$ solve the exact same problem as before, $w_{1}^{L 1}=\max _{z_{1}}\left[A F\left(z_{1}\right)-\zeta z_{1}\right]$, and therefore $z_{1}^{L 1}=z_{0}^{L 1}$. Consequently, levels of productivity and wage in this first layer are the same as in $t=0$. The remaining workers will try to solve problems not yet successfully addressed in layer L1. As stated, a new positive and lower than 1 constant value $h_{L 2}$ will reflect the higher exigency associated to activities in this layer. These workers will solve a fraction of 
the available problems, $\left[F\left(z_{1}^{L 1}+z_{1}\right)-F\left(z_{1}^{L 1}\right)\right] /\left[1-F\left(z_{1}^{L 1}\right)\right]$, and the wage rate will be optimally given by

$w_{1}^{L 2}=\max _{z_{1}}\left[\frac{1}{h_{L 2}} A \frac{F\left(z_{1}^{L 1}+z_{1}\right)-F\left(z_{1}^{L 1}\right)}{1-F\left(z_{1}^{L 1}\right)}-\zeta z_{1}\right]$

The values of output and productivity loss are now aggregate measures that should take into consideration both layers. In concrete,

$$
\begin{gathered}
Y_{1}=A\left(e_{1}^{L 1}+e_{1}^{L 2}\right)=A F\left(z_{1}^{L 1}+z_{1}^{L 2}\right) \\
u_{1}=1-\left(e_{1}^{L 1}+e_{1}^{L 2}\right)=1-F\left(z_{1}^{L 1}+z_{1}^{L 2}\right)
\end{gathered}
$$

where $z_{1}^{L 1}$ and $z_{1}^{L 2}$ are the optimal solutions of the wage maximization problems. Observe, as well, that the amount of productivity relating activities in layer L2, corresponds to $e_{1}^{L 2}=F\left(z_{1}^{L 1}+z_{1}^{L 2}\right)-F\left(z_{1}^{L 1}\right)$.

Again, one considers the exponential distribution to obtain explicit optimal values. The solution of (2) is

$$
z_{1}^{L 2}=-\frac{1}{\lambda} \ln \left(\frac{h_{L 2} \zeta}{\lambda A}\right)
$$

The level of productivity at this second layer comes

$$
e_{1}^{L 2}=\frac{\zeta}{\lambda A}\left(1-\frac{h_{L 2} \zeta}{\lambda A}\right)
$$

and the wage rate is

$$
w_{1}^{L 2}=\frac{A}{h_{L 2}}-\frac{\zeta}{\lambda}\left[1-\ln \left(\frac{h_{\mathrm{L} 2} \zeta}{\lambda A}\right)\right]
$$

Output will be necessarily larger now that one has a second layer of production (and the productivity in the first layer remains exactly the same as in period $t=0)$,

$$
Y_{1}=A\left[1-h_{L 2}\left(\frac{\zeta}{\lambda A}\right)^{2}\right]
$$

Finally, the loss of productivity diminishes since a new layer of production is introduced with the consequent increase in the ability to exploit available technology: 


$$
u_{1}=h_{L 2}\left(\frac{\zeta}{\lambda A}\right)^{2}
$$

As technology $A$ continues to be explored, new layers of production will be added. The problems not yet solved at $t=1,1-F\left(z_{1}^{L 1}+z_{1}^{L 2}\right)$, will be used by workers at $t=2$ to form a new layer L3, following the same kind of mechanism explored above. Again, activities at layer $L 3$ are more demanding than the ones in previous layers of the hierarchy and this is reflected on the higher wage that workers at this new layer will be able to achieve. In this case, one needs to consider a parameter $h_{L 3}<h_{L 2}<1$, to include in the problem for $w_{2}^{L 3}$ in the same way $h_{L 2}$ was introduced in (2). This process is repeated in time and for each added period, an additional layer is also included. As $t$ goes to infinity, output will tend to its potential level, i.e., the setup will converge to a scenario in which $A$ is explored to its full extent.

Asymptotically, the potential of the available technology might be completely fulfilled; however, this requires time to understand how it is possible to address progressively more challenging technical problems. Furthermore, this evolution can be interrupted at any period by a technological breakthrough that introduces a whole new set of problems that restart the process. Creative destruction takes place.

In order to generalize the consecutive layers' analysis, three propositions will be presented. These will make use of the exponential distribution and consider a sequence of values $h_{L T+1}<h_{L T}<\ldots<h_{L 3}<h_{L 2}<1$ for a terminal time period $t=T$ (which eventually is infinite). Moreover, to simplify the presentation of some of the results, it is assumed that each parameter $h$ decays proportionally relatively to the respective last value, i.e., one takes $h_{L \ell+1}=h_{L \ell}(1-\phi)$, with $\phi \in(0,1), \ell \in\{2,3, \ldots, T\}$. Recall that parameters $h$ reflect the working conditions offered by organizations; higher ranked layers will involve harder problems, and only better conditions to develop the productive activity will give the incentive that allows to successfully address sophisticated and complex technological challenges.

Proposition 1. The higher the rank of the assumed layer, the larger will be the wage rate.

Proof. For a given layer $L \ell$ and a time period $t$, the wage maximization problem is

$$
w_{t}^{L \ell}=\max _{z_{t}}\left[\frac{1}{h_{L \ell}} A \frac{F\left(\sum_{i=1}^{\ell-1} z_{t}^{L i}+z_{t}\right)-F\left(\sum_{i=1}^{\ell-1} z_{t}^{L i}\right)}{1-F\left(\sum_{i=1}^{\ell-1} z_{t}^{L i}\right)}-\zeta z_{t}\right]
$$


Under the exponential distribution, the solution for this problem is $z_{t}^{L \ell}=-\frac{1}{\lambda} \ln \left(\frac{h_{L \ell} \zeta}{\lambda A}\right)$. Replacing $z_{t}^{L \ell}$ in the expression for the wage rate,

$$
w_{t}^{L \ell}=\frac{A}{h_{L \ell}}-\frac{\zeta}{\lambda}\left[1-\ln \left(\frac{h_{L \ell} \zeta}{\lambda A}\right)\right]
$$

or, equivalently,

$$
w_{t}^{L \ell}=\frac{A}{(1-\phi)^{\ell-1}}-\frac{\zeta}{\lambda}\left[1-\ln \left(\frac{(1-\phi)^{\ell-1} \zeta}{\lambda A}\right)\right]
$$

To prove the proposition, one just has to consider two consecutive time periods, $\ell$ and $\ell+1$, and verify that $w_{t}^{L \ell+1}>w_{t}^{L \ell}, \forall \ell \in\{1,2, \ldots, T\}$. Solving this inequality, one arrives to the expression $\zeta<\lambda A \frac{\phi}{(1-\phi)^{\ell}} \frac{1}{[-\ln (1-\phi)]}$. Because $\frac{\phi}{(1-\phi)^{\ell}} \frac{1}{[-\ln (1-\phi)]}>1, \forall \ell \in\{1,2, \ldots, T\}, \phi \in(0,1)$, the condition will hold for any value of the underlying parameters

The result in proposition 1 is the direct outcome of assuming values $h_{L \ell}$ translating the idea that higher ranked activities are considered more valuable since they represent the exploitation of more sophisticated problems associated with the available technology. If the society and its organizations want complex problems associated to the available technology to be addressed and solved, they must be prepared to compensate more generously the workers that can approach them. Only with a better wage incentive, workers will incur in the cost of acquiring knowledge to solve harder problems.

Relatively to productivity values, proposition 2 furnishes an additional result.

Proposition 2. The level of productivity at layer $L \ell+1$ is larger than the level of productivity at layer $L \ell$ if the following condition is satisfied:

$$
\zeta<\lambda A \frac{1-\sqrt{\phi}}{(1-\phi)^{\ell}} \vee \zeta>\lambda A \frac{1+\sqrt{\phi}}{(1-\phi)^{\ell}}, \forall \ell=\{1,2, \ldots, T\} .
$$

Proof. For each layer, the productivity value is always the same, independently of the time period that is considered. Namely,

$$
\begin{gathered}
e_{t}^{L 1}=F\left(z_{t}^{L 1}\right) \\
e_{t}^{L 2}=F\left(z_{t}^{L 1}+z_{t}^{L 2}\right)-F\left(z_{t}^{L 1}\right) \\
e_{t}^{L 3}=F\left(z_{t}^{L 1}+z_{t}^{L 2}+z_{t}^{L 3}\right)-F\left(z_{t}^{L 1}+z_{t}^{L 2}\right) \\
\cdots \\
e_{t}^{L T+1}=F\left(\sum_{i=1}^{T} z_{t}^{L i}+z_{t}^{L T+1}\right)-F\left(\sum_{i=1}^{T} z_{t}^{L i}\right) .
\end{gathered}
$$


For the exponential distribution of problems,

$$
\begin{gathered}
e_{t}^{L 1}=1-\frac{\zeta}{\lambda A} \\
e_{t}^{L 2}=\frac{\zeta}{\lambda A}\left\lfloor 1-\frac{(1-\phi) \zeta}{\lambda A}\right\rfloor \\
e_{t}^{L 3}=(1-\phi)\left(\frac{\zeta}{\lambda A}\right)^{2}\left\lfloor 1-\frac{(1-\phi)^{2} \zeta}{\lambda A}\right\rfloor \\
\cdots \\
e_{t}^{L T+1}=(1-\phi)^{\sum_{i=1}^{T}(i-1)} \cdot\left(\frac{\zeta}{\lambda A}\right)^{T}\left[1-\frac{(1-\phi)^{T} \zeta}{\lambda A}\right]
\end{gathered}
$$

In generic terms, one can write the expression for the productivity at any layer $L \ell, \forall \ell \in\{2, \ldots, T\}$ as:

$$
e_{t}^{L \ell}=(1-\phi)^{\substack{i=1 \\ \sum}}(i-1) \cdot\left(\frac{\zeta}{\lambda A}\right)^{\ell-1}\left[1-\frac{(1-\phi)^{\ell-1} \zeta}{\lambda A}\right]
$$

Now, one evaluates condition $e_{t}^{L \ell+1}<e_{t}^{L \ell}$, which is equivalent to

$$
(1-\phi)^{\sum_{i=1}^{\ell}(i-1)} \cdot\left(\frac{\zeta}{\lambda A}\right)^{\ell}\left[1-\frac{(1-\phi)^{\ell} \zeta}{\lambda A}\right]<(1-\phi)^{\sum_{i=1}^{\ell-1}(i-1)} \cdot\left(\frac{\zeta}{\lambda A}\right)^{\ell-1}\left[1-\frac{(1-\phi)^{\ell-1} \zeta}{\lambda A}\right]
$$

Rearranging this last condition, one obtains an equivalent expression, $(1-\phi)^{2 \ell-1} \zeta^{2}-2(1-\phi)^{\ell-1} \lambda A \zeta+(\lambda A)^{2}>0$. The solution of this inequality, when solved in order to the cost parameter $\zeta$, is the condition in the proposition

The proposition has a straightforward corollary.

Corollary 3. Consider a given layer $\ell$. If $\frac{1-\sqrt{\phi}}{(1-\phi)^{\prime}} \geq 1$, then the level of productivity at layer $L \ell+1$ must correspond to a value lower than the level of productivity at layer $L \ell$.

Proof. Recall that condition $\zeta<\lambda A$ is required in order to guarantee that the number of problems relating technology $A$ that are solved remain bounded above zero. In proposition 2 , if it is true that $\frac{1-\sqrt{\phi}}{(1-\phi)^{\prime}} \geq 1$, then the referred condition is satisfied under the inequalities that ensure $e_{t}^{L \ell+1}<e_{t}^{L \ell}$

From proposition 2 and corollary 3, it is straightforward to observe that as $\ell$ becomes larger, the possibility of encountering the relation $e_{t}^{L \ell+1}>e_{t}^{L \ell}$ becomes rarer.

To illustrate the relation between productivity levels in different layers, 
consider a small numerical example. Take $\lambda=1 / 3, A=2, \phi=0.2$. The level of productivity increases from one layer to the next under condition $\lambda A \frac{1-\sqrt{\phi}}{(1-\phi)^{\ell}}<\zeta<\lambda A \frac{1+\sqrt{\phi}}{(1-\phi)^{\ell}}$. For different layers, this corresponds to:

$$
\begin{aligned}
& \ell=1: 0.4607<\zeta<1.206 \\
& \ell=2: 0.5758<\zeta<1.5075 \\
& \ell=3: 0.7198<\zeta<1.8844
\end{aligned}
$$

Constraint $\zeta<\lambda A$ is, in this specific case, $\zeta<0 .(6)$. Thus, for the proposed example, the possibility of rising productivity between layers is restricted to the first two layers; for $\ell=3$, it is no longer possible to observe larger productivity levels for layers of a higher rank. Take $\zeta=0.6$. In this case, $e_{t}^{L 1}=0.1, e_{t}^{L 2}=0.252$, $e_{t}^{L 3}=0.2747, e_{t}^{L 4}=0.2012, e_{t}^{L 5}=0.1086, \ldots$ The production function will reveal, in this circumstance, an initial phase of increasing returns, followed by decreasing returns that lead to a convergence towards $A=2$. Figure 1 presents the evolution of output over time, for the current example.

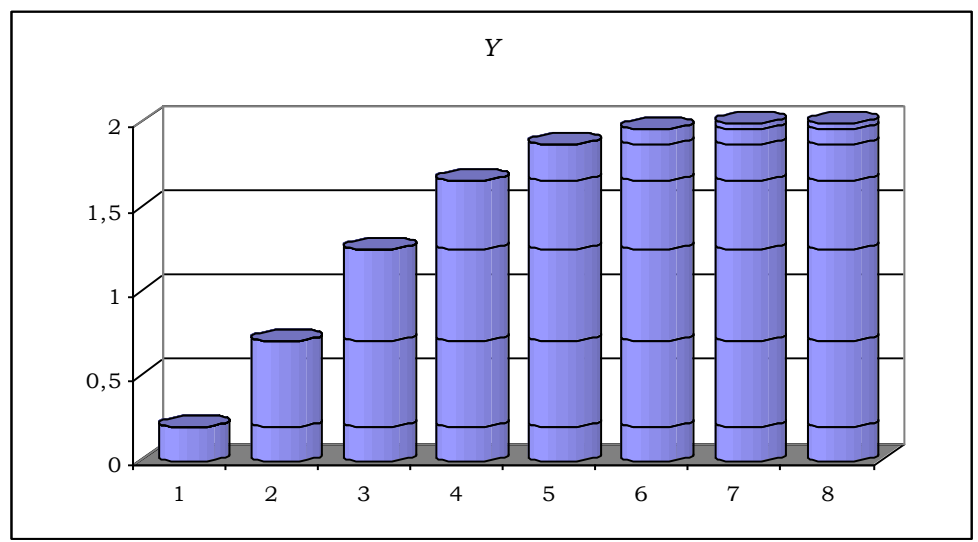

Fig. 1 - Output growth with a constant state of knowledge.

The following proposition addresses the features of the long-run steady-state of the aggregate variables output and productivity loss.

Proposition 4. Assume that technology $A$ is endlessly explored. As $t$ tends to infinity, the output level converges to its potential value, $Y_{P}=A$, and productivity waste falls to zero. 
Proof. At time $t$, the level of output is $Y_{t}=A \sum_{i=1}^{t+1} e_{t}^{L i}$. The sum of all the productivity levels, for each layer, allows to write the output expression as

$$
Y_{t}=A\left[1-(1-\phi)^{\sum_{i=1}^{t+1}(i-1)} \cdot\left(\frac{\zeta}{\lambda A}\right)^{t+1}\right]
$$

Noticing that $\lim _{t \rightarrow \infty}(1-\phi)^{\substack{t+1 \\ i=1}(i-1)}\left(\frac{\zeta}{\lambda A}\right)^{t+1}=0$, it is also true that $\lim _{t \rightarrow \infty} Y_{t}=A$.

Relatively to the measure of productivity loss, this is defined by $u_{t}=1-\sum_{i=1}^{t+1} e_{t}^{L i}$, which is equivalent to

$$
u_{t}=(1-\phi)^{\substack{t+1 \\ \sum_{i=1}(i-1)}} \cdot\left(\frac{\zeta}{\lambda A}\right)^{t+1}
$$

Clearly, $\lim _{t \rightarrow \infty} u_{t}=0$

As time goes to infinity and, therefore, an infinite number of production layers is formed, wages of higher layers also tend to infinity. This does not bring any lack of coherence to the model, because as wages go to infinity, the workers associated with successful generation of output in such layers will tend to zero, which means that the value added to production remains bounded. Under this setting, we may conceive the existence of superstars, i.e., workers with exceptional abilities, that will receive extremely high wages because they are located at highly ranked layers, but that do not have a significant impact in the evolution of the economic system because, when looking at the whole, they are an insignificant number.

\section{Automated Activities}

In this section, the framework of production by layers is reassessed at the light of a new assumption. The assumption relates the idea that, once solved for a first time, some problems associated to technology $A$ no longer require the use of human labor to continue to be solved. Labor resources become useless in basic activities that, once successfully addressed, can be approached without resorting to the labor input: they become automated activities.

To express this new idea, one recovers the precedent analysis at time $t=1$. Recall that at $t=0$ a first layer of activity has solved $z_{0}^{L 1}$ problems associated with technology $A$. The new assumption indicates that a share $\delta \in(0,1)$ of the problems 
solved at $t=0$ are now automatically solved at $t=1$. Thus, at the current period, agents in layer activity $L 1$ will address just a subset of the whole of the problems attached to the technological level, namely the subset $\left[\delta z_{0}^{L 1}, z\right]$. A same type of wage maximization problem is taken into consideration and, therefore, workers at layer L1 will solve

$$
w_{1}^{L 1}=\max _{z_{1}}\left[A \frac{F\left(\delta z_{0}^{L 1}+z_{1}\right)-F\left(\delta z_{0}^{L 1}\right)}{1-F\left(\delta z_{0}^{L 1}\right)}-\zeta z_{1}\right]
$$

The solution of (3) is the same as in the analysis of the previous section, i.e., $z_{1}^{L 1}=-\frac{1}{\lambda} \ln \left(\frac{\zeta}{\lambda A}\right)$. The existence of activities not requiring human intervention does not change, as well, the value of the wage paid to workers in this layer. The change relatively to the first scenario occurs for the contribution of the labor input to production at layer $L 1$. Now this is given by $e_{1}^{L 1}=F\left(\delta z_{0}^{L 1}+z_{1}\right)-F\left(\delta z_{0}^{L 1}\right)$, which is equivalent to

$$
e_{1}^{L 1}=\left(\frac{\zeta}{\lambda A}\right)^{\delta}\left(1-\frac{\zeta}{\lambda A}\right)
$$

Given that $\left(\frac{\zeta}{\lambda A}\right)^{\delta}<1$, under the constraints imposed to parameter values the level of labor productivity is lowered once one considers that some of the activities associated to technology $A$ become automated and no longer require the use of labor resources.

The fact that some activities discard labor is also reflected in the next layer, L2. The wage problem at this second layer is

$$
w_{1}^{L 2}=\max _{z_{1}}\left[\frac{1}{h_{L 2}} A \frac{F\left(\delta z_{0}^{L 1}+z_{1}^{L 1}+z_{1}\right)-F\left(\delta z_{0}^{L 1}+z_{1}^{L 1}\right)}{1-F\left(\delta z_{0}^{L 1}+z_{1}^{L 1}\right)}-\zeta z_{1}\right]
$$

The number of problems solved at layer $L 2$ and the wage of the workers at this layer remain the same as in the benchmark case of the previous section. The level of labor productivity falls to $e_{1}^{L 2}=F\left(\delta z_{0}^{L 1}+z_{1}^{L 1}+z_{1}\right)-F\left(\delta z_{0}^{L 1}+z_{1}^{L 1}\right)$, i.e., to $e_{1}^{L 2}=\left(\frac{\zeta}{\lambda A}\right)^{\delta}\left(\frac{\zeta}{\lambda A}\right)\left(1-\frac{h_{L 2} \zeta}{\lambda A}\right)$.

Thus, by assuming that a set of activities becomes automated, a fraction of the labor productivity at each layer is simply lost. Losing labor productivity does not mean, however, losing output, since the activities that do not need labor are developed exactly as they were before. This implies that output corresponds to the value previously found in the original formulation of the model, which, for $t=1$, is $Y_{1}=A\left[1-h_{L 2}\left(\frac{\zeta}{\lambda A}\right)^{2}\right]$. 
The main consequence of the newly introduced assumption is that now one has two kinds of productivity loss:

1) Short-run productivity loss - this is the productivity loss that exists because technology is not yet fully explored, i.e., it is the type of waste of labor resources dealt with so far. It corresponds to the case $\delta=0$ :

$$
u_{1}^{s}=1-\left.\left(e_{1}^{L 1}+e_{1}^{L 2}\right)\right|_{\delta=0}=h_{L 2}\left(\frac{\zeta}{\lambda A}\right)^{2}
$$

2) Long-run productivity loss - this corresponds to the loss of productivity that occurs as a consequence of activities associated to $A$ that no longer require labor skills to be executed. This second type of waste of labor resources is designated by $u_{1}^{l}$, and it will correspond to the difference between overall productivity loss and short-run productivity loss. The total level of labor productivity waste is straightforward to obtain, $u_{1}=1-\left(e_{1}^{L 1}+e_{1}^{L 2}\right)=1-\left(\frac{\zeta}{\lambda A}\right)^{\delta}\left[1-h_{L 2}\left(\frac{\zeta}{\lambda A}\right)^{2}\right]$; thus, obsolete skills will simply correspond to

$$
u_{1}^{l}=u_{1}-u_{1}^{s}=\left[1-\left(\frac{\zeta}{\lambda A}\right)^{\delta}\right]\left[1-h_{L 2}\left(\frac{\zeta}{\lambda A}\right)^{2}\right]
$$

In order to confirm the value of output in this case, just observe that it will respect to the technology level times the sum of productivity with the share of labor inefficiency that does not respect to loss of production, $Y_{1}=A\left(e_{1}^{L 1}+e_{1}^{L 2}+u_{1}^{l}\right)$.

By adding new layers, the two productivity loss measures will display distinct patterns of evolution as long as the technology level remains at $A$. While $u^{s}$ converges to zero, the share $u^{l}$ will remain, in the long-term, at a positive constant value, what implies that under a steady-state perspective the only way to fight the waste of labor resources is through an education process capable of minimizing skills obsolescence. Before generalizing the analysis to an undefined sequence of time periods, a clearer perception of the mechanics of the economy requires looking at $t=2$.

At $t=2$, again a share $\delta$ of problems solved at $t=1$ will not require labor resources, and thus the relevant set of problems is $\left[\delta\left(z_{1}^{L 1}+z_{1}^{L 2}\right), z\right]$. The wage expressions for the three layers are

$$
w_{2}^{L 1}=\max _{z_{2}}\left[A \frac{F\left[\delta\left(z_{1}^{L 1}+z_{1}^{L 2}\right)+z_{2}\right]-F\left[\delta\left(z_{1}^{L 1}+z_{1}^{L 2}\right)\right]}{1-F\left[\delta\left(z_{1}^{L 1}+z_{1}^{L 2}\right)\right]}-\zeta z_{2}\right]
$$




$$
\begin{gathered}
w_{2}^{L 2}=\max _{z_{2}}\left[\frac{1}{h_{L 2}} A \frac{F\left[\delta\left(z_{1}^{L 1}+z_{1}^{L 2}\right)+z_{2}^{L 1}+z_{2}\right]-F\left[\delta\left(z_{1}^{L 1}+z_{1}^{L 2}\right)+z_{2}^{L 1}\right]}{1-F\left[\delta\left(z_{1}^{L 1}+z_{1}^{L 2}\right)+z_{2}^{L 1}\right]}-\zeta z_{2}\right] \\
w_{2}^{L 3}=\max _{z_{2}}\left[\frac{1}{h_{L 3}} A \frac{F\left[\delta\left(z_{1}^{L 1}+z_{1}^{L 2}\right)+z_{2}^{L 1}+z_{2}^{L 2}+z_{2}\right]-F\left[\delta\left(z_{1}^{L 1}+z_{1}^{L 2}\right)+z_{2}^{L 1}+z_{2}^{L 2}\right]}{1-F\left[\delta\left(z_{1}^{L 1}+z_{1}^{L 2}\right)+z_{2}^{L 1}+z_{2}^{L 2}\right]}-\zeta z_{2}\right]
\end{gathered}
$$

The values of $z_{1}^{L 1}, z_{2}^{L 1}, z_{1}^{L 3}$ and of $w_{2}^{L 1}, w_{2}^{L 2}, w_{2}^{L 3}$ are the same ones as before. The changes are found in the levels of labor productivity at each layer, which are lower than before,

$$
\begin{gathered}
e_{2}^{L 1}=\left[h_{L 2}\left(\frac{\zeta}{\lambda A}\right)^{2}\right]^{\delta}\left(1-\frac{\zeta}{\lambda A}\right) \\
e_{2}^{L 2}=\left[h_{L 2}\left(\frac{\zeta}{\lambda A}\right)^{2}\right]^{\delta} \frac{\zeta}{\lambda A}\left(1-\frac{h_{L 2} \zeta}{\lambda A}\right) \\
e_{2}^{L 3}=\left[h_{L 2}\left(\frac{\zeta}{\lambda A}\right)^{2}\right]^{\delta} h_{L 2}\left(\frac{\zeta}{\lambda A}\right)^{2}\left(1-\frac{h_{L 3} \zeta}{\lambda A}\right)
\end{gathered}
$$

The measure of productivity waste is now $u_{2}=1-\left(e_{2}^{L 1}+e_{2}^{L 2}+e_{2}^{L 3}\right)$, i.e.,

$$
u_{2}=1-\left[h_{L 2}\left(\frac{\zeta}{\lambda A}\right)^{2}\right]^{\delta}\left[1-h_{L 2} h_{L 3}\left(\frac{\zeta}{\lambda A}\right)^{3}\right]
$$

which corresponds to the sum of short-run and long-run labor productivity losses,

$$
\begin{gathered}
u_{2}^{s}=h_{\mathrm{L} 2} h_{\mathrm{L} 3}\left(\frac{\zeta}{\lambda A}\right)^{3} \\
u_{2}^{l}=\left\{1-\left[h_{\mathrm{L} 2}\left(\frac{\zeta}{\lambda A}\right)^{2}\right]^{\delta}\right\}\left[1-h_{\mathrm{L} 2} h_{L 3}\left(\frac{\zeta}{\lambda A}\right)^{3}\right]
\end{gathered}
$$

The description of the results relating $t=2$ ends with the presentation of aggregate output, which is the same as in the case of no skills obsolescence, 


$$
Y_{2}=A\left(e_{2}^{L 1}+e_{2}^{L 2}+e_{2}^{L 3}+u_{2}^{l}\right)=A\left[1-h_{L 2} h_{L 3}\left(\frac{\zeta}{\lambda A}\right)^{3}\right]
$$

Consider now $t=T \geq 2$.

Proposition 5. Results in propositions 1 and 2 continue to hold under the assumption of skills obsolescence.

Proof. For $t=T$, it is assumed that a share of problems solved at $t=T-1$ no longer requires labor resources to be addressed, so that the relevant set of problems relating technology $A$ is $\left[\delta \sum_{i=1}^{T} z_{T-1}^{L i}, z\right]$.

The generic problem that an agent in layer $L \ell, \forall \ell \in\{1,2, \ldots, T\}$, must solve at period $T$ is

$$
w_{T}^{L \ell}=\max _{z_{T}}\left[\frac{1}{h_{L \ell}} A \frac{F\left(\delta \sum_{i=1}^{T} z_{T-1}^{L i}+\sum_{i=1}^{\ell-1} z_{T}^{L i}+z_{T}\right)-F\left(\delta \sum_{i=1}^{T} z_{T-1}^{L i}+\sum_{i=1}^{\ell-1} z_{T}^{L i}\right)}{1-F\left(\sum_{i=1}^{T} z_{T-1}^{L i}+\sum_{i=1}^{\ell-1} z_{T}^{L i}\right)}-\zeta z_{T}\right]
$$

The solution of this problem comes $z_{T}^{L \ell}=-\frac{1}{\lambda} \ln \left(\frac{(1-\phi)^{\ell-1} \zeta}{\lambda A}\right)$ and the wage rate is the same as in the case of no skill obsolescence, $w_{T}^{L \ell}=\frac{A}{h_{L \ell}}-\frac{\zeta}{\lambda}\left[1-\ln \left(\frac{h_{L \ell} \zeta}{\lambda A}\right)\right]$. Thus, as proved under proposition 1 , wages are higher for layers of a superior rank.

The labor productivity level at layer $L \ell$ is

$$
e_{T}^{L \ell}=\left[(1-\phi)^{\sum_{i=1}^{T}(i-1)} \cdot\left(\frac{\zeta}{\lambda A}\right)^{T}\right]^{\delta}(1-\phi)^{\substack{\ell-1 \\ i=1}}(i-1) \cdot\left(\frac{\zeta}{\lambda A}\right)^{\ell-1}\left[1-\frac{(1-\phi)^{\ell-1} \zeta}{\lambda A}\right]
$$

The evaluation of condition $e_{t}^{L \ell+1}<e_{t}^{L \ell}$ for the new productivity values leads exactly to the same double inequality as in proposition 2 . This is true because the first term in the new expression for $e_{T}^{L \ell},\left[(1-\phi)^{\sum_{i=1}^{T}(i-1)} \cdot\left(\frac{\zeta}{\lambda A}\right)^{T}\right]^{\delta}$, is the same for the productivity in every layer, while the remaining terms of the expression compose the value of the productivity rate in the original case, for which $\delta=0$

Next, one addresses the long-term outcomes concerning output and labor productivity loss.

Proposition 6. Consider that no new technological innovation takes place, so that $A$ 
remains the available state of knowledge as $T \rightarrow \infty$. The output level converges to $A$ and labor productivity loss will converge to $u_{T}^{l}$.

Proof. Output is defined by $Y_{T}=A\left(\sum_{i=1}^{T+1} e_{T}^{L i}+u_{T}^{l}\right)$. This is an already known expression, $Y_{T}=A\left[1-(1-\phi)^{\substack{\sum_{i=1}^{2}(i-1) \\ i=1}} \cdot\left(\frac{\zeta}{\lambda A}\right)^{T+1}\right]$. The term after the minus sign converges to zero as time goes to infinity, and therefore, as before, $\lim _{T \rightarrow \infty} Y_{T}=A$.

The productivity loss expression is given by

$$
u_{T}=1-\sum_{i=1}^{T+1} e_{T}^{L i}=1-\left[(1-\phi)^{\substack{T \\ i=1}}(i-1) \cdot\left(\frac{\zeta}{\lambda A}\right)^{T}\right]^{\delta}\left[1-(1-\phi)^{\substack{T+1 \\ i=1}}(i-1) \cdot\left(\frac{\zeta}{\lambda A}\right)^{T+1}\right] .
$$

The labor productivity loss value can be split in the two mentioned categories, namely

and

$$
u_{T}^{s}=(1-\phi)^{\substack{T+1 \\ i=1}(i-1)} \cdot\left(\frac{\zeta}{\lambda A}\right)^{T+1}
$$

$$
u_{T}^{l}=\left\{1-\left[(1-\phi)^{\sum_{i=1}^{T}(i-1)} \cdot\left(\frac{\zeta}{\lambda A}\right)^{T}\right]^{\delta}\right\}\left[1-(1-\phi)^{\sum_{i=1}^{T+1}(i-1)} \cdot\left(\frac{\zeta}{\lambda A}\right)^{T+1}\right]
$$

The short-run productivity loss will converge to zero in the long-term, and all the waste in labor productivity will, asymptotically, correspond to skills obsolescence,

$$
u_{T}=u_{T}^{l}=1-\left[(1-\phi)^{\sum_{i=1}^{T}(i-1)} \cdot\left(\frac{\zeta}{\lambda A}\right)^{T}\right]^{\delta}
$$

\section{The Preferences of a Representative Agent}

The previous arguments were silent on what determines parameter $\delta$. It was claimed that a share of the problems solved in the last period no longer need labor resources to continue to be solved, however this share arose arbitrarily in the analysis. In what follows, it is assumed that the choices of a representative household will lead to the emergence of an optimal share of problems to which no labor is allocated to.

The representative agent will solve a static optimization problem. Her 
objective is to maximize utility, and the utility function will contain two arguments, consumption and the long-run loss of productivity; it takes the form $U\left(C, u^{l}\right)$, where $C$ represents consumption. As it should be evident, utility increases with consumption, $\partial U / \partial C>0$, and decreases with skills obsolescence, $\partial U / \partial u^{l}<0$. The function must also involve decreasing marginal utility concerning both arguments. The following functional form will be taken: $U=\ln C-m \ln u^{l}$, with $m>0$ the agent's preference for low skills obsolescence, relative to high consumption levels.

The control variable in this optimization problem will be a share $\tau \in(0,1)$ that indicates how much of the income generated by the productive activity is allocated to consumption and how much of the same income is directed to investment in education in order to prevent labor obsolescence. In particular, let this parameter represent the income diverted from consumption towards schooling. With this share in mind, consumption might be represented as $C=(1-\tau) Y$. The problem solved by the representative agent will be, at each date $t, \underset{\tau}{\operatorname{Max} U} \mathrm{.}^{2}$

The representative agent chooses a share $\tau$ that maximizes utility of a high consumption and of a low rate of labor productivity loss. There is a trade-off: to lower the obsolescence of skills, a fraction of income must be used in education, and thus it becomes unavailable for households to spend. The problem is subject to a constraint that indicates how resources allocated to education, $\tau Y$, may be used to mitigate job destruction, $\delta \sum_{i=1}^{T} z_{T-1}^{L i}$. This constraint will correspond to a continuous function that obeys the following conditions: $\tau Y=0 \Rightarrow \delta=\delta^{\max } ; \tau Y \rightarrow \infty \Rightarrow \delta=0$. These conditions say that in the absence of education effort $(\tau=0)$, the share of problems that will have no attached labor units will correspond to the maximum share $\delta^{\max } \in(\delta, 1)$. On the other extreme, if an infinite quantity of resources is allocated to schooling, then long-run losses on the use of labor resources are completely eliminated.

The elimination of the exclusion of the labor force from participating in the productive process in this case implies that activities that would require no labor to be developed will continue to be developed by some of the workers in the economy, without this adding anything to the capacity of the economy to generate output. This poses a question: why would the representative agent want to preserve skills if activities can be developed without human effort? The answer

${ }^{2}$ It is possible to transform this into an intertemporal control problem of the type $\max _{\tau} \sum_{t=0}^{\infty} \beta^{t} U$, with $\beta \in(0,1)$ a discount factor. However, this does not change the results because the constraint to this problem, to be considered below, is intrinsically static. 
will be given in section 5 , where one will consider a change in the technological paradigm; once a new state of technology emerges, only the share of the workforce that was capable of maintaining its skills intact and that was participating in the productive activity in the moment the technical paradigm shift occurs will be able to continue to produce. Thus, society has an objective advantage in keeping workers inside the productive process; if this does not occur, some productive resources will be forever lost, and labor productivity becomes compromised as new technological states emerge.

A rule that satisfies the previous conditions and that fits the requirements of the analysis is:

$\delta \sum_{i=1}^{T} z_{T-1}^{L i}=-\frac{1}{\lambda} \ln \left[1+\frac{\exp \left(-\lambda \delta^{\max } \sum_{i=1}^{T} z_{T-1}^{L i}\right)-1}{1+\tau Y_{T}}\right]$

For $t=1$, the relevant education constraint is

$$
\delta z_{0}^{L 1}=-\frac{1}{\lambda} \ln \left[1+\frac{\exp \left(-\lambda \delta^{\max } z_{0}^{L 1}\right)-1}{1+\tau Y_{1}}\right]
$$

For the known value of $z_{0}^{L 1}$ this is equivalent to

$$
\left(\frac{\zeta}{\lambda A}\right)^{\delta}=1-\frac{1-\left(\frac{\zeta}{\lambda A}\right)^{\delta^{\max }}}{1+\tau Y_{1}}
$$

Remember that long-run productivity loss at $t=1$ is $u_{1}^{l}=\left[1-\left(\frac{\zeta}{\lambda A}\right)^{\delta}\right]\left[1-h_{L 2}\left(\frac{\zeta}{\lambda A}\right)^{2}\right]$ . Replace the expression for $\left(\frac{\zeta}{\lambda A}\right)^{\delta}$ in the value of $u^{l}$, in order to rewrite the utility function,

$U_{1}=\ln (1-\tau) Y_{1}-m \ln \left\{\frac{1-\left(\frac{\zeta}{\lambda A}\right)^{\delta^{\max }}}{1+\tau Y_{1}}\left[1-h_{L 2}\left(\frac{\zeta}{\lambda A}\right)^{2}\right]\right\}$

The maximization of (6) requires the computation of a first-order optimality condition:

$$
\frac{\partial U_{1}}{\partial \tau}=0 \Rightarrow \tau=\frac{m Y_{1}-1}{(1+m) Y_{1}}
$$


Observe that $\tau$ will be positive only if $m>1 / Y_{1}$. To avoid the possibility of a negative share of investment in education, one sets $\tau=\max \left\{0 ; \frac{m Y_{1}-1}{(1+m) Y_{1}}\right\}$.

The optimal value of $\tau$ can be replaced in the term involving $\delta$,

$$
\left(\frac{\zeta}{\lambda A}\right)^{\delta}=1-\left[1-\left(\frac{\zeta}{\lambda A}\right)^{\delta^{\max }}\right] \frac{1+m}{m\left(1+Y_{1}\right)}
$$

The previous value might, then, be replaced in the expressions of productivity and productivity loss of the previous section, in order to obtain the corresponding values when there is an explicit concern with preserving skills.

Again, the generalization of the presented reasoning is straightforward. At $t=2$, the problem faced by the representative agent is

$$
\begin{gathered}
\operatorname{Max}_{\tau}\left[\ln (1-\tau) Y_{2}-m \ln u_{2}^{l}\right] \\
\text { subject to : } \delta\left(z_{1}^{L 1}+z_{1}^{L 2}\right)=-\frac{1}{\lambda} \ln \left[1+\frac{\exp \left(-\lambda \delta^{\max }\left(z_{1}^{L 1}+z_{1}^{L 2}\right)\right)-1}{1+\tau Y_{1}}\right]
\end{gathered}
$$

The solution of the problem is similar to the one in the case $t=1$, that is, $\tau_{2}=\max \left\{0 ; \frac{m Y_{2}-1}{(1+m) Y_{2}}\right\}$. Now, the relevant term to compute productivity values is

$$
\left[h_{L 2}\left(\frac{\zeta}{\lambda A}\right)^{2}\right]^{\delta}=1-\left\{1-\left[h_{L 2}\left(\frac{\zeta}{\lambda A}\right)^{2}\right]^{\text {max }}\right\} \frac{1+m}{m\left(1+Y_{2}\right)}
$$

The analysis for $t=T$ yields the following general results,

Proposition 7. A representative agent, simultaneously concerned with current consumption and current preservation of skills at $t=T$, will adopt an education investment share $\tau_{T}=\max \left\{0 ; \frac{m Y_{T}-1}{(1+m) Y_{T}}\right\}$, that does not change the amount of output, $Y_{T}$, but that lowers the long-run productivity loss from

$$
u_{T}^{l}=\left\{1-\left[(1-\phi)^{\sum_{i=1}^{T}(i-1)} \cdot\left(\frac{\zeta}{\lambda A}\right)^{T}\right]^{\delta^{\max }}\right\}\left[1-(1-\phi)^{\substack{T_{+1}+1 \\ i=1}}(i-1)\left(\frac{\zeta}{\lambda A}\right)^{T+1}\right]
$$

to

$$
u_{T}^{l}=\left\{1-\left[(1-\phi)^{\sum_{i=1}^{T}(i-1)} \cdot\left(\frac{\zeta}{\lambda A}\right)^{T}\right]^{\delta^{\max }}\right\} \frac{1+m}{m\left(1+Y_{T}\right)}\left[1-(1-\phi)^{\substack{T+1 \\ i_{i=1}(i-1)}} \cdot\left(\frac{\zeta}{\lambda A}\right)^{T+1}\right]
$$


Proof. For $t=T$, the representative agent solves problem $\operatorname{Max} U_{T}=\left[\ln (1-\tau) Y_{T}-m \ln u_{T}^{l}\right]$. The maximization of $U_{T}$ will be subject to (5). The solution of the problem is obtained after computation of $\frac{\partial U_{T}}{\partial \tau}=0$. The result is $\tau_{T}=\frac{m Y_{T}-1}{(1+m) Y_{T}}$. A positive share of investment in education exists only for $m>1 / Y_{T}$. Avoiding a negative investment in education implies presenting the respective expression as $\tau_{T}=\max \left\{0 ; \frac{m Y_{T}-1}{(1+m) Y_{T}}\right\}$. Because $Y$ is not dependent on the amount of labor that is allocated to activities that can be completely automated, the level of output remains the same that was found in the precedent section.

Given the constraint (5), and the optimal share of schooling investment, one can replace, in the expression of the productivity loss value, the term $\left[(1-\phi)^{\sum_{i=1}^{T}(i-1)} \cdot\left(\frac{\zeta}{\lambda A}\right)^{T}\right]^{\delta}$ by

$$
1-\left\{1-\left[(1-\phi)^{\sum_{i=1}^{T}(i-1)} \cdot\left(\frac{\zeta}{\lambda A}\right)^{T}\right]^{\delta^{\max }}\right\} \frac{1+m}{m\left(1+Y_{T}\right)}
$$

This allows to write the long-run productivity loss as in the last expression of the proposition

Note that if no investment in education is implemented, $\tau=0$ (what will occur for a relatively low preference for a small level of skills obsolescence, i.e., $m \leq 1 / Y_{T}$ ), the long-run productivity loss value is kept at the level that takes into account the maximum possible share of automated problems $\delta^{\max }$. This value is lowered when share $\tau$ is positive. Comparing the two values of $u_{T}^{l}$ in the proposition, for $\tau=0$ and $\tau>0$ respectively, one sees that in the second case the loss of productivity is a share $\frac{1+m}{m\left(1+Y_{T}\right)}$ of the loss of productivity in the first case.

A last result concerns the steady-state level of productivity loss.

Proposition 8. Under the optimal choice of the representative agent, in the case in which $m>1 / A$, the productivity loss level converges to $u_{T}=\frac{1+m}{m(1+A)}$ as $T \rightarrow \infty$. For $m \leq 1 / A$, the productivity loss goes to 1 as time goes to infinity.

Proof. Proposition 6 revealed that $\lim _{T \rightarrow \infty} Y_{T}=A$ independently of the value of $\delta$. This is true because the level of output is independent of how many jobs are kept regarding activities that do not need labor any longer. Production takes place, in this case, whether people work or not. Thus, in the long-term one must distinguish between two situations: $m>1 / A$ and $m \leq 1 / A$. In the first situation, it is optimal to adopt a positive percentage of investment in education $\tau=\frac{m A-1}{(1+m) A}$ (this is the 
previously computed optimal education investment share, adapted to the case where $T \rightarrow \infty)$. Replacing share $\tau$ in the expression of the productivity loss value or the expression of the long-run productivity loss value (which are the same, since $u^{s} \rightarrow 0$ ), one gets $\lim _{T \rightarrow \infty} u_{T}=\frac{1+m}{m(1+A)}$. In the second situation, the share of investment in education will be zero, no concern will exist with preserving skills and therefore

$$
u_{T}^{l}=\left\{1-\left[(1-\phi)^{\sum_{i=1}^{T}(i-1)} \cdot\left(\frac{\zeta}{\lambda A}\right)^{T}\right]^{\delta^{\max }}\right\}\left\{1-(1-\phi)^{\sum_{i=1}^{T+1}(i-1)} \cdot\left(\frac{\zeta}{\lambda A}\right)^{T+1}\right\}
$$

and

$$
u_{T}=1-\left[(1-\phi)^{\sum_{i=1}^{T}(i-1)} \cdot\left(\frac{\zeta}{\lambda A}\right)^{T}\right]^{\delta^{\max }}\left[1-(1-\phi)^{\sum_{i=1}^{T+1}(i-1)} \cdot\left(\frac{\zeta}{\lambda A}\right)^{T+1}\right]
$$

will both converge to 1 . In this scenario, all activities will be automated in the longterm and, thus, the loss of productivity will be maximum

\section{Technological Progress}

So far, the process of implementation of a technology was characterized without including the possibility of innovation, that is, of a change in the state of the available techniques. The eventuality of a technological breakthrough is now considered. The main implication of innovation is that there is a radical shift of workers from the first technology to the second and this implies that the process of adding layers to the economic activity starts all over again. One will consider that the emergence of a new technology occurs endogenously; the representative agent will include the benefits and costs of technology adoption in its welfare function and when the benefits of adopting the new technology exceed the respective costs, the old technology is left behind and a new process of technology adoption initiates.

The framework of technological transition is as follows. An economy that exploits technology $A$ has the possibility of evolving to a new technology $A^{\prime}=A(1+\gamma), \gamma>0$. The costs associated to the change of technology paradigm are given by function $c(A)=A \psi \gamma^{2}, \psi>0$. The representative agent will compare the level of utility as assumed so far, $U_{t}=\ln (1-\tau) Y_{t}-m \ln u_{t}^{l}$, with the level of utility that would be achieved if the new technology had already been implemented, i.e.,

$$
U_{t}^{\prime}=\ln (1-\tau) Y_{t}^{\prime}-m \ln u_{t}^{l^{\prime}}-c(A)
$$

The values of $Y_{t}^{\prime}$ and $u_{t}^{l^{\prime}}$ correspond to output and long-run productivity loss for the new technological level. 
The optimization problem of the representative agent can be solved to encounter the optimal value of the investment in education, and this allows to present the maximum levels of utility in the following form:

$$
U_{t}=\ln \frac{1+Y_{t}}{1+m}-m \ln \frac{(1+m) Y_{t}}{m\left(1+Y_{t}\right)}\left\{1-\left[\frac{A-Y_{t}}{(1-\phi)^{t} \frac{\zeta}{\lambda}}\right]^{\delta^{\max }}\right\}
$$

and

$$
U_{t}^{\prime}=\ln \frac{1+Y_{t}^{\prime}}{1+m}-m \ln \frac{(1+m) Y_{t}^{\prime}}{m\left(1+Y_{t}^{\prime}\right)}\left\{1-\left[\frac{A(1+\gamma)-Y_{t}^{\prime}}{(1-\phi)^{t} \frac{\zeta}{\lambda}}\right]^{\delta^{\max }}\right\}-c(A)
$$

Assume that the values of parameters are such that $U_{0}>U_{0}^{\prime}$, and thus that it does not pay to adopt immediately the new technology. As time passes, the costs of technology adoption remain constant while output under the new technology becomes progressively larger when compared with the income the economy withdraws from the use of the initial state of technology. Therefore there will be a turning point in time $t=T$, in which $U^{\prime}$ becomes larger than $U$. At this point, the society chooses to irreversibly evolve to the next technological stage. A relevant consequence of a change in the technological paradigm is that a share of workers already left out the productive process under the precedent technological state will not be able, as well, to apply for a job under the new state of technology. This longrun productivity loss will now remain an unrecoverable loss. This share is

$$
u_{T-1}^{l}=\left\{1-\left[(1-\phi)^{\sum_{i=1}^{T-1}(i-1)} \cdot\left(\frac{\zeta}{\lambda A}\right)^{T-1}\right]^{\delta}\right\}\left[1-(1-\phi)^{\sum_{i=1}^{T}(i-1)} \cdot\left(\frac{\zeta}{\lambda A}\right)^{T}\right]
$$

In the first period of the exploitation of $A^{\prime}$, values of productivity, productivity loss and income will be computed as in the case of the first technology, i.e.,

$$
\begin{gathered}
\left(e_{0}^{L 1}\right)^{\prime}=1-\frac{\zeta}{\lambda A^{\prime}} \\
\left(u_{0}\right)^{\prime}=\frac{\zeta}{\lambda A^{\prime}} \\
\left(Y_{0}\right)^{\prime}=A^{\prime}-\frac{\zeta}{\lambda}
\end{gathered}
$$

The technology is better and the costs of knowledge acquisition and the shape 
of the distribution function do not change. Thus, the economy will generate more output. Innovation is synonymous of a greater potential to grow. The exhibited productivity and productivity loss values take into account just the amount of workers that have evolved to the new technological state and, therefore, they do not really represent the true productivity and productivity loss levels. One can obtain these by taking the following expressions:

- Productivity in the first period of the use of the new technology:

$\left.e_{0}^{L 1}\right|_{A^{\prime}}=\frac{\left(e_{0}^{L 1}\right)^{\prime}}{1+u_{T-1}^{l}} ;$

- Loss of productivity in the first period of the use of the new technology:

$\left.u_{0}\right|_{A^{\prime}}=\frac{\left(u_{0}\right)^{\prime}+u_{T-1}^{l}}{1+u_{T-1}^{l}}$.

To evaluate the immediate impact of a technology adoption on the productivity level, one must compare $\left.u_{0}\right|_{A^{\prime}}$ with $u_{T-1}^{l}$. The loss of productivity falls instantly with the adoption of the new technology if $\left.u_{0}\right|_{A^{\prime}}<u_{T-1}^{l}$, which is equivalent to $\left(u_{0}\right)^{\prime}<\left(u_{T-1}^{l}\right)^{2}$.

Considering additional periods after the adoption of the new technology,

Proposition 9. Assume a new technology is adopted at $t=T$ and let $m>1 / A^{\prime}$. Hereafter, output converges to $A^{\prime}$ and the measure of loss of productivity converges to

$$
\left.u_{\infty}\right|_{A^{\prime}}=\frac{1+m+m\left(1+A^{\prime}\right) u_{T-1}^{l}}{m\left(1+A^{\prime}\right)\left(1+u_{T-1}^{l}\right)} \text {. }
$$

The long-run productivity loss is lower, in the long-term, under the adoption of the new technology comparatively with the case in which the technology is not adopted, when the following condition holds: $\left(u_{T-1}^{l}\right)^{2}>\frac{1+m}{m\left(1+A^{\prime}\right)}$.

Proof. Output will converge to the state of technology because potential output continues to correspond to the level of the available state of knowledge. The productivity loss value $u_{T-1}^{l}$ does not have any influence over $Y^{\prime}$ because the solution of the wage problem for every $t$ above $T$ is independent of any productivity waste that remained locked in the previous technology level. At each date $t$, a level of productivity $\sum_{i=1}^{t+1}\left(e_{t}^{L i}\right)^{\prime}$ allows to produce 
$Y_{t}^{\prime}=A^{\prime}\left[1-(1-\phi)^{\sum_{i-1}^{t+1}(i-1)} \cdot\left(\frac{\zeta}{\lambda A}\right)^{t+1}\right]$, which converges to $A^{\prime}$ as time evolves.

The productivity loss that is generated under the new technology, $\left(u_{t}\right)^{\prime}$, corresponds to a level that is similar to the one found for $A$. This converges, under the concern with skill obsolescence to $\left(u_{\infty}\right)^{\prime}=\frac{1+m}{m(1+A)}$. To this loss of skills, we must add the loss of productivity respecting to $u_{T-1}^{l}$, and therefore $\left.u_{\infty}\right|_{A^{\prime}}=\frac{\left(u_{\infty}\right)^{\prime}+u_{T-1}^{l}}{1+u_{T-1}^{T}}$. Rearranging, one obtains the expression in the proposition. The comparison between the labor productivity loss that will end up by prevailing and the one that existed before the technological change requires evaluating condition $\left.u_{\infty}\right|_{A^{\prime}}<u_{T-1}^{l}$. The inequality in the proposition, which is equivalent to this, assures that it is favorable to adopt the new technology, from the point of view of achieving a low waste of labor resources

The meaningful point of the analysis of technological change is that we can compare, from a quantitative point of view, productivity that is lost and productivity that is gained when a new technology is introduced. Unskilled people will not have the ability to ever recover a job position under the new technology, but the remaining workers will have additional wage incentives to become productive. Which force dominates depends on the extent of the technological innovation and on how many people were excluded from the productive activity at the time of the change in the production methods.

Evidently, the analysis could be extended to successive changes in the state of technology. One might argue that as new technologies are adopted, one will accumulate layers of unproductive people that never return to the market in search for a new job. The counter argument is that if one is considering such an extensive time interval that allows to assume systematic technological changes, then one should also assume that in this interval generations are renewed, i.e., those who lost their working skills are retired and new and better prepared workers enter the labor market with the capacity to handle the most advanced technological challenges.

\section{Conclusion}

This paper has taken a framework of production by layers and adapted it to explain how productivity evolves in time. On a growth perspective, one can think of productivity waste essentially as the outcome of two forces: on one hand, new technologies involve problems that take time to be explored and efficiently managed; problems not yet solved will have correspondence on a share of the workforce displaying low levels of productivity. On the other hand, some human 
activities relating the technology in use turn obsolete; these correspond to tasks that become fully automated once the productive system understands well how to make use of them. The first type of productivity loss is a short-run productivity loss that is reduced and even eliminated as the economy achieves a good capacity of handling the techniques. The second type of productivity loss is a long-run productivity loss since it will correspond to the share of workers that are unable to generate value because their skills are no longer needed to continue to produce.

A representative agent, representing the preferences of the society as a whole, may dislike the exclusion of part of the labor force from the productive process (because, in fact, this has significant consequences on the ability of the economy to create value once a technological paradigm shift occurs) and might find it useful to sacrifice some of the potential current consumption in order to empower workers with skills that allow them to continue active members on the process of wealth creation. In this framework, it is possible to determine an optimal rate of investment in education, i.e., a share of resources to allocate to schooling that maximize utility given some function representing the agent's preferences. The possibility of technological progress was also addressed in this context. A change to a better technology leaves behind the group of those who could not preserve their skills under the old technology; however, the improved technology stimulates problem solving by the remaining part of the workforce, allowing in this way to raise productivity. The conflict between these two processes will determine whether the new technology enhances the net rise of the contribution of the labor input to production or if the opposite occurs.

\section{References}

Acemoglu, D.; G. Gancia and F. Zilibotti (2012). "Competing Engines of Growth: Innovation and Standardization." Journal of Economic Theory, vol. 147, pp. 570601.

Aghion, P. and P. Howitt (1992). "A Model of Growth Through Creative Destruction." Econometrica, vol. 60, pp. 323-351.

Apergis, N.; C. Economidou and I. Filippidis (2008). "Innovation, Technology Transfer and Labor Productivity Linkages: Evidence from a Panel of Manufacturing Industries." Review of World Economics, vol. 144, pp. 491-508.

Cosar, A.K. (2011). "Human Capital, Technology Adoption and Development." the B.E. Journal of Macroeconomics, vol. 11, issue 1, 5 pages.

Francois, P. and H. Lloyd-Ellis (2003). "Animal Spirits Through Creative Destruction." American Economic Review, vol. 93, pp. 530-550.

Garicano, L. and E. Rossi-Hansberg (2010). "Organizing Growth." Journal of 
Economic Theory, vol. 144, pp. 2477-2502.

Grossman, G.M. and E. Helpman (1991). "Quality Ladders in the Theory of Growth." Review of Economic Studies, vol. 59, pp. 43-61.

Hodgson, G.M. (1998). "Evolutionary and Competence-Based Theories of the Firm." Journal of Economic Studies, vol. 25, pp. 25-56.

Nissim, B.D. (2007). "Economic Growth and Its Effects on Income Distribution." Journal of Economic Studies, vol. 34, pp. 42-58.

Phillips, K.L. (2012). "Specialization and Market Development as Engines of Growth." Journal of Economic Studies, vol. 39, pp. 590-603.

Razzak, W.A. (2007). "Explaining the Gaps in Labor Productivity in Some Developed Countries: New Zealand, Australia, the United States and Canada, 1988-2004." Applied Econometrics and International Development, vol. 7, issue 2.

Serletis, A. and G. Feng (2006). "Productivity Trends in the United States." Journal of Economic Studies, vol. 33, pp. 320-335.

Teles, V.K. and J. Andrade (2008). "Public Investment in Basic Education and Economic Growth." Journal of Economic Studies, vol. 35, pp. 352-364.

Thesmar, D. and M. Thoenig (2000). "Creative Destruction and Firm Organization Choice." Quarterly Journal of Economics, vol. 115, pp. 1201-1237. 